\title{
Quasiparticle interaction in nuclear matter with chiral three-nucleon forcesa
}

\author{
J. W. Holt, N. Kaiser, and W. Weise \\ Physik Department, Technische Universität München, D-85747 Garching, Germany
}

\begin{abstract}
We derive the effective interaction between two quasiparticles in symmetric nuclear matter resulting from the leading-order chiral three-nucleon force. We restrict our study to the $L=0,1$ Landau parameters of the central quasiparticle interaction computed to first order. We find that the three-nucleon force provides substantial repulsion in the isotropic spin- and isospin-independent component $F_{0}$ of the interaction. This repulsion acts to stabilize nuclear matter against isoscalar density oscillations, a feature which is absent in calculations employing low-momentum two-nucleon interactions only. We find a rather large uncertainty for the nuclear compression modulus $\mathcal{K}$ due to a sensitive dependence on the low-energy constant $c_{3}$. The effective nucleon mass $M^{*}$ on the Fermi surface, as well as the nuclear symmetry energy $\beta$, receive only small corrections from the leading-order chiral three-body force. Both the anomalous orbital $g$-factor $\delta g_{l}$ and the Landau-Migdal parameter $g_{N N}^{\prime}$ (characterizing the spin-isospin response of nuclear matter) decrease with the addition of three-nucleon correlations. In fact, $\delta g_{l}$ remains significantly smaller than its value extracted from experimental data, whereas $g_{N N}^{\prime}$ still compares well with empirical values. The inclusion of the three-nucleon force results in relatively small $p$-wave $(L=1)$ components of the central quasiparticle interaction, thus suggesting an effective interaction of short range.
\end{abstract}

\footnotetext{
${ }^{a}$ Work supported in part by BMBF, GSI and by the DFG cluster of excellence: Origin and Structure of the Universe.
} 


\section{INTRODUCTION}

In a recent publication [1] we have studied the quasiparticle interaction in isospin-symmetric nuclear matter employing realistic chiral nucleon-nucleon (NN) interactions. The second-order calculation performed in ref. [1] explored the order-by-order convergence of the perturbative expansion for the quasiparticle interaction as well as the scale dependence resulting from the renormalization group evolution [2, 3] of the underlying two-body interaction. Although good agreement was found for a number of nuclear observables, such as the effective mass $M^{*}$ at the Fermi surface, the nuclear symmetry energy $\beta$ and the spin-isospin response encoded in the parameter $g_{N N}^{\prime}$, both the compression modulus $\mathcal{K}$ of isospin-symmetric nuclear matter and the anomalous orbital $g$-factor $\delta g_{l}$ were found to differ appreciably from empirical values extrapolated from collective excitations of finite nuclei. Most seriously, the compression modulus $\mathcal{K}$ of nuclear matter (encoded in the Fermi liquid parameter $F_{0}$ ) was found to be negative at both first- and second-order across a wide range of cutoff scales.

Previous studies [4-8] have suggested that interactions induced by the polarization of the medium (first studied by Babu and Brown for the case of liquid ${ }^{3} \mathrm{He}$ [9]) provide sufficient repulsion to stabilize nuclear matter against isoscalar density fluctuations. In fact, already the leading-order contribution to the Babu-Brown induced interaction is quite repulsive [1], though not enough to achieve stability at normal nuclear matter saturation density when employing chiral or low-momentum NN interactions. For such potentials, numerous calculations [10 13] of the equation of state have revealed the necessity of three-nucleon forces in driving saturation toward the empirical density $\rho_{0}=0.16 \mathrm{fm}^{-3}$ and energy per particle $\bar{E}=-16 \mathrm{MeV}$.

To improve the microscopic description of the quasiparticle interaction, we compute in the present work the first-order (perturbative) contribution to the $L=0,1$ Landau parameters resulting from the leading-order chiral three-nucleon force. Previous work [14] performed within the framework of chiral effective field theory included explicit $\Delta$-isobar degrees of freedom in a calculation of the isotropic central Fermi liquid parameters. In the present study we employ the high-precision Idaho $\mathrm{N}^{3} \mathrm{LO}$ chiral NN interaction [15] together with the $\mathrm{N}^{2} \mathrm{LO}$ chiral threenucleon force [16], which subsumes certain processes involving virtual $\Delta$-isobar excitations considered in ref. [14]. The additional repulsion provided by the leading-order three-nucleon force is expected to play an important role for the Landau parameter $F_{0}$, but the extent to which other nuclear observables respond to additional three-nucleon correlations has not been studied systematically in calculations employing high-precision NN potentials.

The present paper is organized as follows. In Section II we briefly review Landau's theory 
of normal Fermi liquids and discuss the connection between physical observables and various Fermi liquid parameters. We then derive analytical expressions for the first-order contribution to the $L=0,1$ central Landau parameters arising from the leading-order chiral three-nucleon interaction. In section III we present numerical results for the density and scale dependence of the quasiparticle interaction supplemented by the second-order contributions of ref. [1]. The latter were derived from the Idaho $\mathrm{N}^{3} \mathrm{LO}$ chiral two-nucleon interaction as well as from the (universal) low-momentum NN interaction $V_{\text {low-k }}$. We end with a summary and an outlook.

\section{NUCLEAR QUASIPARTICLE INTERACTION}

\section{A. Landau parameters and nuclear observables}

Fermi liquid theory was introduced by Landau in the 1950's [17] to describe the properties of strongly interacting normal many-fermion systems at low temperatures. The low-energy excitations about the ground state are long-lived quasiparticles that retain certain features of non-interacting (independent) particles but have modified dynamical properties such an effective mass and an effective magnetic moment. Fermi liquid theory has been used to describe a wide variety of quantum many-body systems, including various types of conductors at low temperatures, liquid ${ }^{3} \mathrm{He}$, nuclear matter, neutron matter and also finite nuclei [18 20$]$.

The quasiparticle interaction $\mathcal{F}\left(\vec{p}_{1} \sigma_{1} \tau_{1} ; \vec{p}_{2} \sigma_{2} \tau_{2}\right)$ encodes bulk equilibrium and transport properties of a Fermi liquid. It arises at second order in the expansion of the energy density in terms of powers of the quasiparticle distribution function $\delta n_{\vec{p} \sigma \tau}$ :

$$
\delta \mathcal{E}=\sum_{\vec{p}, \sigma, \tau} \epsilon_{\vec{p}} \delta n_{\vec{p} \sigma \tau}+\frac{1}{2} \sum_{\substack{\vec{p}_{1} \sigma_{1} \tau_{1} \\ \vec{p}_{2} \sigma_{2} \tau_{2}}} \mathcal{F}\left(\vec{p}_{1} \sigma_{1} \tau_{1} ; \vec{p}_{2} \sigma_{2} \tau_{2}\right) \delta n_{\vec{p}_{1} \sigma_{1} \tau_{1}} \delta n_{\vec{p}_{2} \sigma_{2} \tau_{2}}+\cdots
$$

where $\epsilon_{\vec{p}}$ is the single-particle energy and higher-order quasiparticle correlations are neglected. The central part of the quasiparticle interaction in spin- and isospin-saturated nuclear matter has the form

$$
\mathcal{F}\left(\vec{p}_{1}, \vec{p}_{2}\right)=f\left(\vec{p}_{1}, \vec{p}_{2}\right)+f^{\prime}\left(\vec{p}_{1}, \vec{p}_{2}\right) \vec{\tau}_{1} \cdot \vec{\tau}_{2}+\left[g\left(\vec{p}_{1}, \vec{p}_{2}\right)+g^{\prime}\left(\vec{p}_{1}, \vec{p}_{2}\right) \vec{\tau}_{1} \cdot \vec{\tau}_{2}\right] \vec{\sigma}_{1} \cdot \vec{\sigma}_{2}
$$

where $\vec{\sigma}_{1,2}$ and $\vec{\tau}_{1,2}$ denote the spin and isospin operators of the two nucleons on the Fermi surface $\left|\vec{p}_{1}\right|=\left|\vec{p}_{2}\right|=k_{F}$. More generally, the quasiparticle interaction can include (noncentral) tensor components which modify the stability conditions of nuclear matter [21] and are necessary for understanding the magnetic susceptibility [22] and the response of nuclear matter to weak probes [23, 24]. For two quasiparticles on the Fermi surface, the remaining angular 
dependence of their interaction can be expanded in Legendre polynomials of $\cos \theta=\hat{p}_{1} \cdot \hat{p}_{2}$ :

$$
X\left(\vec{p}_{1}, \vec{p}_{2}\right)=\sum_{L=0}^{\infty} X_{L}\left(k_{F}\right) P_{L}(\cos \theta)
$$

where $X$ represents $f, f^{\prime}, g$, or $g^{\prime}$, and the angle $\theta$ is related to the relative momentum $p=$ $\frac{1}{2}\left|\vec{p}_{1}-\vec{p}_{2}\right|$ through the relation $p=k_{F} \sin (\theta / 2)$. The coefficients of the expansion in eq.(3) are referred to as the Fermi liquid parameters (FLPs). It is conventional to introduce dimensionless FLPs by multiplying by the density of states at the Fermi surface, $N_{0}=2 M^{*} k_{F} / \pi^{2}$, where $M^{*}$ is the nucleon effective mass and $k_{F}$ is the Fermi momentum, leading to

$$
\mathcal{F}\left(\vec{p}_{1}, \vec{p}_{2}\right)=\frac{1}{N_{0}} \sum_{L=0}^{\infty}\left[F_{L}+F_{L}^{\prime} \vec{\tau}_{1} \cdot \vec{\tau}_{2}+\left(G_{L}+G_{L}^{\prime} \vec{\tau}_{1} \cdot \vec{\tau}_{2}\right) \vec{\sigma}_{1} \cdot \vec{\sigma}_{2}\right] P_{L}(\cos \theta)
$$

For short-range interactions the expansion in (4) is typically rapidly converging such that only a few constants characterize the dynamics of low-energy excitations. Moreover, individual parameters evaluated at the equilibrium Fermi momentum $k_{F}=1.33 \mathrm{fm}^{-1}$ are related to properties of the quasiparticles and the bulk nuclear medium:

$$
\begin{aligned}
\text { Quasiparticle effective mass : } \frac{M^{*}}{M_{N}} & =1+F_{1} / 3, \\
\text { In-medium orbital } g \text {-factor: } \quad g_{l} & =\frac{1+\tau_{3}}{2}+\frac{F_{1}^{\prime}-F_{1}}{6\left(1+F_{1} / 3\right)} \tau_{3}, \\
\text { Compression modulus : } \quad \mathcal{K} & =\frac{3 k_{F}^{2}}{M^{*}}\left(1+F_{0}\right), \\
\text { Isospin asymmetry energy : } \quad \beta & =\frac{k_{F}^{2}}{6 M^{*}}\left(1+F_{0}^{\prime}\right), \\
\text { Spin-isospin response : } \quad g_{N N}^{\prime} & =\frac{4 M_{N}^{2}}{g_{\pi N}^{2} N_{0}} G_{0}^{\prime},
\end{aligned}
$$

where the anomalous orbital $g$-factor $\delta g_{l}$ is given by

$$
\delta g_{l}=\frac{F_{1}^{\prime}-F_{1}}{6\left(1+F_{1} / 3\right)}
$$

and $g_{\pi N} \simeq 13.2$ is the strong $\pi N$ coupling constant. Spin observables, though largely unconstrained experimentally, receive significant contributions from the (non-central) tensor Fermi liquid parameters [22].

Recently, we have carried out systematic calculations [1] of the quasiparticle interaction in nuclear matter to second order in many-body perturbation theory employing chiral and low-momentum NN interactions. The first- and second-order contributions, shown diagrammatically in Fig. 1, have the form

$$
\mathcal{F}^{(1)}\left(\vec{p}_{1} s_{1} t_{1} ; \vec{p}_{2} s_{2} t_{2}\right)=\left\langle\vec{p}_{1} s_{1} t_{1} ; \vec{p}_{2} s_{2} t_{2}|\bar{V}| \vec{p}_{1} s_{1} t_{1} ; \vec{p}_{2} s_{2} t_{2}\right\rangle \equiv\langle 12|\bar{V}| 12\rangle
$$




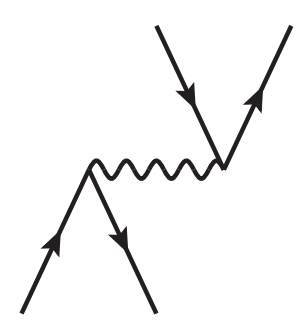

(a)

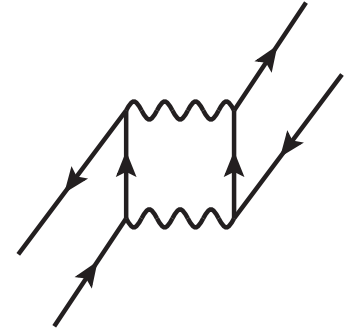

(b)

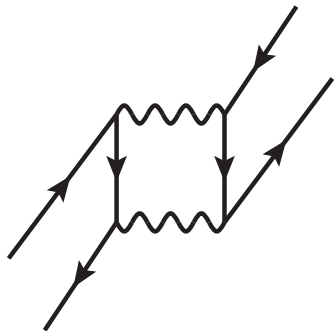

$(c)$

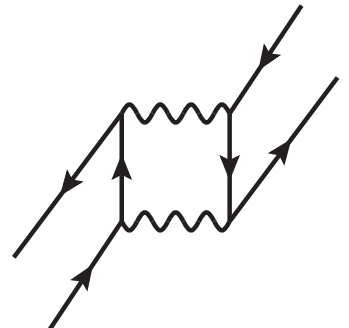

$(d)$

FIG. 1. First- and second-order diagrammatic contributions to the quasiparticle interaction. The wavy lines symbolize the two-nucleon interaction.

and

$$
\begin{aligned}
\mathcal{F}^{(2)}\left(\vec{p}_{1} s_{1} t_{1} ; \vec{p}_{2} s_{2} t_{2}\right) & =\frac{1}{2} \sum_{34} \frac{|\langle 12|\bar{V}| 34\rangle|^{2}\left(1-n_{3}\right)\left(1-n_{4}\right)}{\epsilon_{1}+\epsilon_{2}-\epsilon_{3}-\epsilon_{4}} \\
& +\frac{1}{2} \sum_{34} \frac{|\langle 12|\bar{V}| 34\rangle|^{2} n_{3} n_{4}}{\epsilon_{3}+\epsilon_{4}-\epsilon_{1}-\epsilon_{2}}-2 \sum_{34} \frac{|\langle 13|\bar{V}| 24\rangle|^{2} n_{3}\left(1-n_{4}\right)}{\epsilon_{1}+\epsilon_{3}-\epsilon_{2}-\epsilon_{4}},
\end{aligned}
$$

where the quantity $\bar{V}=\left(1-P_{12}\right) V$ denotes the antisymmetrized two-body potential and $n_{j}=\theta\left(k_{F}-\left|\vec{k}_{j}\right|\right)$ is the usual zero-temperature Fermi distribution. These calculations revealed the importance of second-order diagrams in raising the quasiparticle effective mass from $M^{*} \simeq$ $0.7 M_{N}$ to $M^{*} \simeq M_{N}$ (both lying in the phenomenological range $M^{*} / M=0.7-1.0[25$, 26] ) as well as increasing the symmetry energy from $\beta \simeq(21-25) \mathrm{MeV}$ to $\beta \simeq(31-33) \mathrm{MeV}$ (where only the second-order result lies within the range of empirical values $\beta=(30-36) \mathrm{MeV}[27,28])$. The isotropic spin-isospin Landau parameter $G_{0}^{\prime}$ increases at second order, with the effect that $g_{N N}^{\prime}$ changes from a value of around $0.50-0.57$ to about $0.67-0.77$, the latter being within the range $g_{N N}^{\prime}=0.6-0.7$ favored by fits to giant Gamow-Teller resonances in heavy nuclei [29] 31 ]. Despite these encouraging results, the Fermi liquid parameter $F_{0}$ remained well below -1 , giving rise to a negative compression modulus $\mathcal{K}$ and a corresponding instability of nuclear matter against density fluctuations in the vicinity of saturation density $\rho_{0}=0.16 \mathrm{fm}^{-3}$. Additionally, the anomalous orbital $g$-factor decreased from $\delta g_{l} \simeq 0.3$ to $\delta g_{l} \simeq 0.1$, which is significantly less than the empirical value $\delta g_{l}=0.20-0.26$ extracted from giant dipole resonances [32]. This feature followed almost entirely from the dramatic increase in the effective mass $M^{*}$ at second order. An improved microscopic description of the quasiparticle interaction may require the consistent implementation of chiral three-nucleon forces. As a first step in this program, we compute here the first-order contribution to the quasiparticle interaction from the $\mathrm{N}^{2} \mathrm{LO}$ chiral three-body force.

According to eq.(1) the quasiparticle interaction is obtained by functionally differentiating 
the energy density twice with respect to the quasiparticle distribution functions. For a general three-nucleon force, the Hartree-Fock contribution to the energy density is given by

$$
\mathcal{E}_{3 N}^{(1)}=\frac{1}{6} \operatorname{tr}_{\sigma_{1} \tau_{1}} \operatorname{tr}_{\sigma_{2} \tau_{2}} \operatorname{tr}_{\sigma_{3} \tau_{3}} \int \frac{d^{3} k_{1}}{(2 \pi)^{3}} \frac{d^{3} k_{2}}{(2 \pi)^{3}} \frac{d^{3} k_{3}}{(2 \pi)^{3}} n_{\vec{k}_{1}} n_{\vec{k}_{2}} n_{\vec{k}_{3}}\left\langle 123\left|\bar{V}_{3 N}\right| 123\right\rangle,
$$

where $\bar{V}_{3 N}=V_{3 N}\left(1-P_{12}-P_{23}-P_{13}+P_{12} P_{23}+P_{13} P_{23}\right)$ denotes the fully antisymmetrized threenucleon interaction and $n_{\vec{k}_{j}}=\theta\left(k_{F}-\left|\vec{k}_{j}\right|\right)+(2 \pi)^{3} \delta^{3}\left(\vec{k}_{j}-\vec{p}_{j}\right) \delta n_{\vec{p}_{j} \sigma_{j} \tau_{j}}$. Functionally differentiating twice with respect to the two quasiparticle distribution functions then leaves an effective twobody interaction containing a single (loop) integral over the filled Fermi sea of nucleons.

The three-nucleon force employed in the present work is the $\mathrm{N}^{2} \mathrm{LO}$ chiral three-nucleon interaction [16], which consists of three components. First, there is a two-pion exchange component

$$
V_{3 N}^{(2 \pi)}=\sum_{i \neq j \neq k} \frac{g_{A}^{2}}{8 f_{\pi}^{4}} \frac{\vec{\sigma}_{i} \cdot \vec{q}_{i} \vec{\sigma}_{j} \cdot \vec{q}_{j}}{\left({\overrightarrow{q_{i}}}^{2}+m_{\pi}^{2}\right)\left({\overrightarrow{q_{j}}}^{2}+m_{\pi}^{2}\right)} F_{i j k}^{\alpha \beta} \tau_{i}^{\alpha} \tau_{j}^{\beta},
$$

where $g_{A}=1.29, f_{\pi}=92.4 \mathrm{MeV}, m_{\pi}=138 \mathrm{MeV}$ (average pion mass) and $\overrightarrow{q_{i}}$ denotes difference between the final and initial momenta of nucleon $i$. The quantity

$$
F_{i j k}^{\alpha \beta}=\delta^{\alpha \beta}\left(-4 c_{1} m_{\pi}^{2}+2 c_{3} \vec{q}_{i} \cdot \vec{q}_{j}\right)+c_{4} \epsilon^{\alpha \beta \gamma} \tau_{k}^{\gamma} \vec{\sigma}_{k} \cdot\left(\vec{q}_{i} \times \vec{q}_{j}\right)
$$

involves three terms proportional to the low-energy constants $c_{1}, c_{3}$ and $c_{4}$, respectively. The summation $\sum_{i \neq j \neq k}$ runs over the six permutations of three nucleons. The one-pion exchange component of the three-nucleon interaction is proportional to the low-energy constant $c_{D}$ :

$$
V_{3 N}^{(1 \pi)}=-\sum_{i \neq j \neq k} \frac{g_{A} c_{D}}{8 f_{\pi}^{4} \Lambda_{\chi}} \frac{\vec{\sigma}_{j} \cdot \vec{q}_{j}}{{\overrightarrow{q_{j}}}^{2}+m_{\pi}^{2}} \vec{\sigma}_{i} \cdot \vec{q}_{j} \vec{\tau}_{i} \cdot \vec{\tau}_{j},
$$

and finally the three-nucleon contact interaction introduces the low-energy constant $c_{E}$ :

$$
V_{3 N}^{(\mathrm{ct})}=\sum_{i \neq j \neq k} \frac{c_{E}}{2 f_{\pi}^{4} \Lambda_{\chi}} \vec{\tau}_{i} \cdot \vec{\tau}_{j}
$$

where $\Lambda_{\chi}=700 \mathrm{MeV}$ sets a natural scale.

The low-energy constants of $V_{3 N}^{(2 \pi)}$ appear already in the two-pion exchange contribution to the nucleon-nucleon interaction and can therefore be fit to NN scattering phase shifts. The analysis of the Nijmegen group [33] resulted in the values $c_{1}=-0.76 \mathrm{GeV}^{-1}, c_{3}=-4.78 \mathrm{GeV}^{-1}$, and $c_{4}=3.96 \mathrm{GeV}^{-1}$, while Entem and Machleidt [15] obtain $c_{1}=-0.81 \mathrm{GeV}^{-1}, c_{3}=-3.2 \mathrm{GeV}^{-1}$, and $c_{4}=5.4 \mathrm{GeV}^{-1}$. The low-energy constants $c_{D}$ and $c_{E}$ of the mid- and short-range chiral three-nucleon interaction can be constrained by properties of nuclear few-body systems. In the present work we employ two versions of $c_{D}$ and $c_{E}$ obtained by fitting the binding energies of $A=3$ nuclei together with the half-life of ${ }^{3} \mathrm{H}$ with the result [34]:

$$
c_{D}=-0.20, \quad c_{E}=-0.205
$$




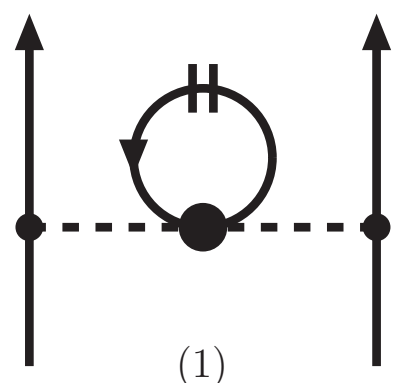

$(1)$

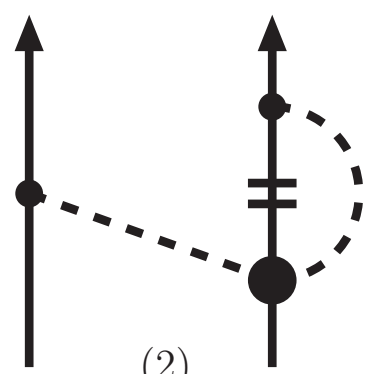

$(2)$

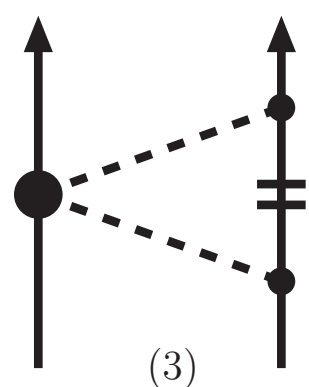

(3)

FIG. 2. Diagrammatic contributions to the quasiparticle interaction in symmetric nuclear matter generated from the two-pion exchange three-nucleon force. The short double-line symbolizes summation over the filled Fermi sea of nucleons. Reflected diagrams of (2) and (3) are not shown.

or by fitting the binding energies of ${ }^{3} \mathrm{H}$ and ${ }^{4} \mathrm{He}[11]$ :

$$
c_{D}=-2.062, \quad c_{E}=-0.625 .
$$

In the first set the leading-order three-body force (with $c_{1,3,4}$ coefficients of Entem and Machleidt) was used together with the Idaho $\mathrm{N}^{3} \mathrm{LO}$ chiral two-nucleon interaction, while in the second set the authors employed the Nijmegen low-energy constants $c_{1,3,4}$ together with the low-momentum NN interaction $V_{\text {low-k }}$ at the resolution scale $\Lambda=2.1 \mathrm{fm}^{-1}$. Employing these two versions of the chiral three-nucleon force (combined with two-nucleon interactions at different scales) provides a means for assessing theoretical errors at this order in the perturbative expansion.

\section{B. Diagrammatic calculation}

We begin by considering the isotropic $(L=0)$ Fermi liquid parameters of the central quasiparticle interaction. They are obtained by angle-averaging the (in-medium) effective interaction [35]:

$$
\begin{aligned}
\mathcal{F}_{0}\left(k_{f}\right) & =\frac{1}{(4 \pi)^{2}} \int d \Omega_{1} d \Omega_{2}\left\langle\vec{p}_{1}, \vec{p}_{2}\left|V_{N N}^{\mathrm{med}}\right| \vec{p}_{1}, \vec{p}_{2}\right\rangle \\
& =f_{0}\left(k_{f}\right)+g_{0}\left(k_{f}\right) \vec{\sigma}_{1} \cdot \vec{\sigma}_{2}+f_{0}^{\prime}\left(k_{f}\right) \vec{\tau}_{1} \cdot \vec{\tau}_{2}+g_{0}^{\prime}\left(k_{f}\right) \vec{\sigma}_{1} \cdot \vec{\sigma}_{2} \vec{\tau}_{1} \cdot \vec{\tau}_{2}
\end{aligned}
$$

where $\left|\vec{p}_{1} \vec{p}_{2}\right\rangle$ denotes an antisymmetrized two-nucleon state. Both quasiparticle momenta $\vec{p}_{1}$ and $\vec{p}_{2}$ lie on the Fermi surface so that $\left|\vec{p}_{1}\right|=\left|\vec{p}_{2}\right|=k_{f}$. There are six topologically distinct one-loop diagrams, shown in Figs. 2 and 3 , contributing to the effective interaction $V_{N N}^{\text {med }}$. Using the abbreviations $\boldsymbol{\sigma}=\vec{\sigma}_{1} \cdot \vec{\sigma}_{2}$ and $\boldsymbol{\tau}=\vec{\tau}_{1} \cdot \vec{\tau}_{2}$, we find for the crossed term of diagram (1) in Fig. 2 the following contribution

$$
\mathcal{F}_{0}\left(k_{f}\right)^{(\text {med,1) }}=(3-\boldsymbol{\sigma})(3-\boldsymbol{\tau}) \frac{g_{A}^{2} m_{\pi}^{3}}{(6 \pi)^{2} f_{\pi}^{4}}\left\{\frac{\left(2 c_{1}-c_{3}\right) u^{3}}{1+4 u^{2}}-c_{3} u^{3}+\left(c_{3}-c_{1}\right) \frac{u}{2} \ln \left(1+4 u^{2}\right)\right\},
$$



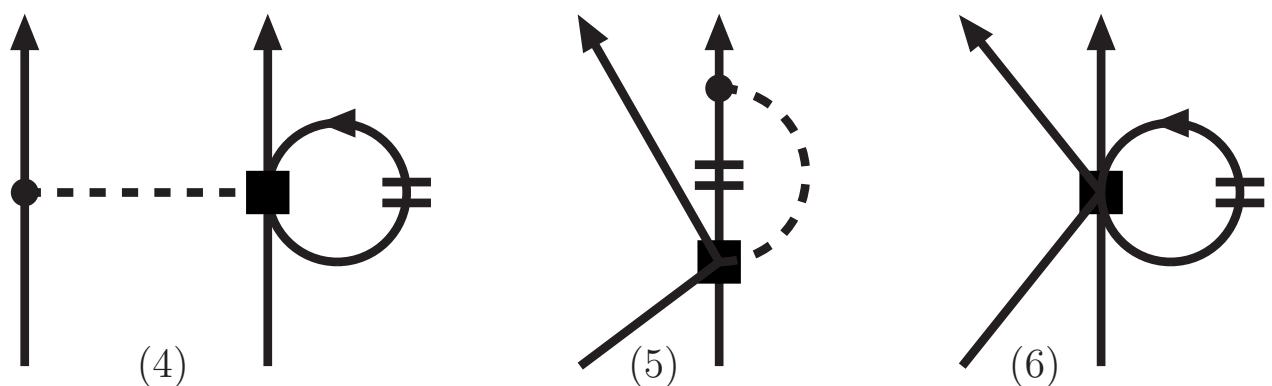

FIG. 3. Diagrammatic contributions to the quasiparticle interaction in symmetric nuclear matter generated by one-pion exchange and contact three-nucleon forces. Reflected diagrams of (4) and (5) are not shown. The last two diagrams (5) and (6) contribute only to the $L=0$ Landau parameters.

with $u=k_{F} / m_{\pi}$. In this diagram, the two pions carry equal momenta and therefore the term in eq.(11) proportional to $c_{4}$ does not contribute. Moreover, the direct term (with zero momentum transfer) of this pion self-energy correction vanishes trivially. Analogously, only the crossed term from the one-pion exchange vertex correction (diagram (2) in Fig. 2) is nonzero with the analytical result:

$$
\begin{aligned}
\mathcal{F}_{0}\left(k_{f}\right)^{(\operatorname{med}, 2)} & =(3-\boldsymbol{\sigma})(3-\boldsymbol{\tau}) \frac{g_{A}^{2} m_{\pi}^{3}}{(24 \pi)^{2} f_{\pi}^{4}}\left\{\frac{3 c_{1}}{8 u^{5}}\left[4 u^{2}-\ln \left(1+4 u^{2}\right)\right]\right. \\
& \times\left[8 u^{4}+4 u^{2}-\left(1+4 u^{2}\right) \ln \left(1+4 u^{2}\right)\right] \\
& +c_{3}\left[\frac{2}{u^{2}}\left(4 u^{2}-\ln \left(1+4 u^{2}\right)\right) \arctan 2 u+\frac{48 u^{4}+16 u^{2}+3}{64 u^{7}} \ln ^{2}\left(1+4 u^{2}\right)\right. \\
& \left.+\frac{12 u^{4}-16 u^{6}-30 u^{2}-9}{24 u^{5}} \ln \left(1+4 u^{2}\right)+\frac{20 u^{3}}{3}-11 u+\frac{1}{u}+\frac{3}{4 u^{3}}\right] \\
& +c_{4}\left[\frac{4}{u^{2}}\left(\ln \left(1+4 u^{2}\right)-4 u^{2}\right) \arctan 2 u+\frac{3+16 u^{2}-48 u^{4}}{64 u^{7}} \ln ^{2}\left(1+4 u^{2}\right)\right. \\
& \left.\left.+\frac{80 u^{6}+12 u^{4}-30 u^{2}-9}{24 u^{5}} \ln \left(1+4 u^{2}\right)-\frac{28 u^{3}}{3}+13 u+\frac{1}{u}+\frac{3}{4 u^{3}}\right]\right\} .
\end{aligned}
$$

The Pauli-blocked two-pion exchange component (diagram (3) in Fig. 2) has both a nonvanishing direct and crossed term. Their sum takes the form

$$
\begin{aligned}
\mathcal{F}_{0}\left(k_{f}\right)^{(\text {med,3) }} & =\frac{g_{A}^{2} m_{\pi}^{3}}{(4 \pi)^{2} f_{\pi}^{4}}\left\{24\left(c_{3}-c_{1}\right) u-8 c_{3} u^{3}+\left(3 c_{3}-4 c_{1}\right) \frac{3}{u} \ln \left(1+4 u^{2}\right)\right. \\
& +6\left(6 c_{1}-5 c_{3}\right) \arctan 2 u+(3-\boldsymbol{\sigma})(3-\boldsymbol{\tau}) \frac{c_{4}}{9} \int_{0}^{u} d x\left(Y^{2}-X^{2}\right) \\
& \left.+(1+\boldsymbol{\sigma})(1+\boldsymbol{\tau}) \int_{0}^{u} d x\left[3 c_{1} Z^{2}+\frac{c_{3}}{2}\left(X^{2}+2 Y^{2}\right)\right]\right\},
\end{aligned}
$$


where the auxiliary functions $X, Y$, and $Z$ arising from Fermi sphere integrals over a pion propagator read

$$
\begin{aligned}
& X(u, x)=2 x-\frac{1}{2 u} \ln \frac{1+(u+x)^{2}}{1+(u-x)^{2}}, \\
& Y(u, x)=\frac{5 x^{2}-3 u^{2}-3}{4 x}+\frac{4 x^{2}+3\left(1+u^{2}-x^{2}\right)^{2}}{16 u x^{2}} \ln \frac{1+(u+x)^{2}}{1+(u-x)^{2}}, \\
& Z(u, x)=1+\frac{x^{2}-u^{2}-1}{4 u x} \ln \frac{1+(u+x)^{2}}{1+(u-x)^{2}} .
\end{aligned}
$$

Note that the direct term contributes only to the spin- and isospin-independent Landau parameter $F_{0}$.

There are two diagrammatic contributions from the mid-range one-pion exchange chiral three-nucleon force, labeled as (4) and (5) in Fig. 3. The crossed term from the $1 \pi$-exchange vertex correction (diagram (4)) leads to the contribution

$$
\mathcal{F}_{0}\left(k_{f}\right)^{(\operatorname{med}, 4)}=(3-\boldsymbol{\sigma})(3-\boldsymbol{\tau}) \frac{g_{A} c_{D} m_{\pi}^{3}}{(24 \pi)^{2} f_{\pi}^{4} \Lambda_{\chi}}\left[4 u^{3}-u \ln \left(1+4 u^{2}\right)\right]
$$

and the sum of direct and crossed terms from diagram (5) yields

$$
\mathcal{F}_{0}\left(k_{f}\right)^{(\mathrm{med}, 5)}=(3-\boldsymbol{\sigma}-\boldsymbol{\tau}-\boldsymbol{\sigma} \boldsymbol{\tau}) \frac{g_{A} c_{D} m_{\pi}^{3}}{(4 \pi)^{2} f_{\pi}^{4} \Lambda_{\chi}}\left\{\frac{2 u^{3}}{3}-u+\arctan 2 u-\frac{1}{4 u} \ln \left(1+4 u^{2}\right)\right\} .
$$

Finally, the three-nucleon contact term generates a contribution proportional to the nuclear density $\rho=2 k_{F}^{3} / 3 \pi^{2}$. The sum of direct and crossed terms reads

$$
\mathcal{F}_{0}\left(k_{f}\right)^{(\mathrm{med}, 6)}=(\boldsymbol{\sigma}+\boldsymbol{\tau}+\boldsymbol{\sigma} \boldsymbol{\tau}-3) \frac{c_{E} k_{f}^{3}}{4 \pi^{2} f_{\pi}^{4} \Lambda_{\chi}} .
$$

We note that for all three-body contributions the spin-spin and isospin-isospin components of the quasiparticle interaction are equal, $g_{0}=f_{0}^{\prime}$.

The $p$-wave $(L=1)$ Fermi liquid parameters are obtained by weighting the angular integrals by the first Legendre polynomial $3 P_{1}(\cos \theta)=3 \hat{p}_{1} \cdot \hat{p}_{2}$ :

$$
\begin{aligned}
\mathcal{F}_{1}\left(k_{f}\right) & =\frac{3}{(4 \pi)^{2}} \int d \Omega_{1} d \Omega_{2}\left(\hat{p}_{1} \cdot \hat{p}_{2}\right)\left\langle\vec{p}_{1}, \vec{p}_{2}\left|V_{N N}^{\text {med }}\right| \vec{p}_{1}, \vec{p}_{2}\right\rangle \\
& =f_{1}\left(k_{f}\right)+g_{1}\left(k_{f}\right) \vec{\sigma}_{1} \cdot \vec{\sigma}_{2}+f_{1}^{\prime}\left(k_{f}\right) \vec{\tau}_{1} \cdot \vec{\tau}_{2}+g_{1}^{\prime}\left(k_{f}\right) \vec{\sigma}_{1} \cdot \vec{\sigma}_{2} \vec{\tau}_{1} \cdot \vec{\tau}_{2} .
\end{aligned}
$$

After this weighting, the two short-range contributions (diagrams (5) and (6) in Fig. 3) to the quasiparticle interaction vanish. We provide the expressions for the four remaining pieces below. The crossed term from the pion self-energy correction takes the form

$$
\begin{aligned}
\mathcal{F}_{1}\left(k_{f}\right)^{(\text {med }, 1)} & =(3-\boldsymbol{\sigma})(3-\boldsymbol{\tau}) \frac{g_{A}^{2} m_{\pi}^{3}}{48 \pi^{2} f_{\pi}^{4}}\left\{\frac{\left(2 c_{1}-c_{3}\right) u}{1+4 u^{2}}+\left(6 c_{1}-5 c_{3}\right) u\right. \\
& \left.+\left[2\left(c_{3}-c_{1}\right) u+\frac{3 c_{3}-4 c_{1}}{2 u}\right] \ln \left(1+4 u^{2}\right)\right\} .
\end{aligned}
$$


The crossed term from the one-pion exchange vertex correction reads

$$
\begin{aligned}
\mathcal{F}_{1}\left(k_{f}\right)^{(\text {med,2) }} & =(3-\boldsymbol{\sigma})(3-\boldsymbol{\tau}) \frac{g_{A}^{2} m_{\pi}^{3}}{(16 \pi)^{2} f_{\pi}^{4}}\left\{\frac{c_{1}}{4 u^{7}}\left[4 u^{2}-\left(1+2 u^{2}\right) \ln \left(1+4 u^{2}\right)\right]\right. \\
& \times\left[8 u^{4}+4 u^{2}-\left(1+4 u^{2}\right) \ln \left(1+4 u^{2}\right)\right] \\
& +\frac{c_{3}}{3 u^{4}}\left[4\left(4 u^{2}-\left(1+2 u^{2}\right) \ln \left(1+4 u^{2}\right)\right) \arctan 2 u\right. \\
& +\frac{96 u^{6}+80 u^{4}+22 u^{2}+3}{32 u^{5}} \ln ^{2}\left(1+4 u^{2}\right) \\
& +\frac{56 u^{6}-32 u^{8}-60 u^{4}-48 u^{2}-9}{12 u^{3}} \ln \left(1+4 u^{2}\right) \\
& \left.+\frac{4 u^{5}}{3}\left(7-4 u^{2}\right)-14 u^{3}+5 u+\frac{3}{2 u}\right] \\
& +\frac{c_{4}}{3 u^{4}}\left[8\left(\left(1+2 u^{2}\right) \ln \left(1+4 u^{2}\right)-4 u^{2}\right) \arctan 2 u\right. \\
& +\frac{3+22 u^{2}-16 u^{4}-96 u^{6}}{32 u^{5}} \ln ^{2}\left(1+4 u^{2}\right) \\
& +\frac{160 u^{8}-136 u^{6}-60 u^{4}-48 u^{2}-9}{12 u^{3}} \ln \left(1+4 u^{2}\right) \\
& \left.\left.-\frac{4 u^{5}}{3}\left(4 u^{2}+17\right)+34 u^{3}+5 u+\frac{3}{2 u}\right]\right\} .
\end{aligned}
$$

The crossed term from Pauli-blocked two-pion exchange is given by

$$
\begin{aligned}
\mathcal{F}_{1}\left(k_{f}\right)^{(\mathrm{med}, 3)} & =\frac{g_{A}^{2} m_{\pi}^{3}}{(4 \pi)^{2} f_{\pi}^{4}} \int_{0}^{u} d x\left\{(3-\boldsymbol{\sigma})(3-\boldsymbol{\tau}) \frac{c_{4}}{9}\left(3 X_{b}^{2}+2 X_{c}^{2}-3 X_{a}^{2}\right)\right. \\
& \left.+(1+\boldsymbol{\sigma})(1+\boldsymbol{\tau})\left[3 c_{1}\left(Z_{a}^{2}+2 Z_{b}^{2}\right)+\frac{c_{3}}{2}\left(3 X_{a}^{2}+6 X_{b}^{2}+4 X_{c}^{2}\right)\right]\right\}
\end{aligned}
$$

with auxiliary functions

$$
\begin{aligned}
Z_{a}(u, x) & =\frac{x}{u}+\frac{u^{2}-x^{2}-1}{4 u^{2}} \ln \frac{1+(u+x)^{2}}{1+(u-x)^{2}} \\
Z_{b}(u, x) & =\frac{x^{2}-3 u^{2}-3}{4 u x}+\frac{4\left(1+u^{2}+u^{4}\right)-\left(u^{2}+x^{2}-1\right)^{2}}{16 u^{2} x^{2}} \ln \frac{1+(u+x)^{2}}{1+(u-x)^{2}}, \\
X_{a}(u, x) & =\frac{1}{u}-\frac{u^{2}+x^{2}+1}{4 u^{2} x} \ln \frac{1+(u+x)^{2}}{1+(u-x)^{2}}, \\
X_{b}(u, x) & =\frac{1}{8 u x^{2}}\left[2 x^{2}\left(1+u^{2}\right)-3 x^{4}-3\left(1+u^{2}\right)^{2}\right] \\
& +\frac{1+u^{2}+x^{2}}{32 u^{2} x^{3}}\left[3\left(1+u^{2}-x^{2}\right)^{2}+4 x^{2}\right] \ln \frac{1+(u+x)^{2}}{1+(u-x)^{2}}, \\
X_{c}(u, x) & =\frac{1}{8 u x^{2}}\left[3\left(1+u^{2}\right)^{2}-8 u^{2} x^{2}-3 x^{4}\right] \\
& +\frac{3}{32 u^{2} x^{3}}\left(x^{2}-1-u^{2}\right)\left[\left(1+u^{2}+x^{2}\right)^{2}-4 u^{2} x^{2}\right] \ln \frac{1+(u+x)^{2}}{1+(u-x)^{2}}
\end{aligned}
$$

Finally, the only nonvanishing term from the mid-range three-nucleon force is the crossed term 
from diagram (4) in Fig. 3, which reads

$$
\mathcal{F}_{1}\left(k_{f}\right)^{(\mathrm{med}, 4)}=(3-\boldsymbol{\sigma})(3-T) \frac{g_{A} c_{D} m_{\pi}^{3}}{384 \pi^{2} f_{\pi}^{4} \Lambda_{\chi}}\left\{4 u-\left(2 u+\frac{1}{u}\right) \ln \left(1+4 u^{2}\right)\right\} .
$$

Again, one observes that for all contributions, $f_{1}^{\prime}=g_{1}$.

This completes the first-order calculation of the $L=0,1$ Landau parameters arising from the $\mathrm{N}^{2} \mathrm{LO}$ chiral three-nucleon force. A good check of the formulas in eqs.(17)-(35) is given by their Taylor expansions in $k_{F}$. The leading terms are of the form: $c_{E} k_{F}^{3}, c_{D} k_{F}^{5}, c_{1} k_{F}^{5}, c_{3,4} k_{F}^{7}$. Note that full consistency with the second-order calculation in ref. [1] would require the inclusion of the subleading $\mathrm{N}^{3} \mathrm{LO}$ chiral three-body force (recently derived in ref. [36]). Investigations along these lines are in progress.

\section{RESULTS}

In this section we study the density-dependence of the $L=0,1$ Landau parameters derived in the previous section from the leading-order chiral three-nucleon interaction. The values of the five low-energy constants occuring at this order have significant uncertainties, and we employ two different sets which have been fit to reproduce properties of $A=3,4$ nuclei. These contributions are then combined with the results of ref. [1] for the second-order quasiparticle interaction computed with the corresponding two-nucleon interactions. The impact on various nuclear observables is discussed.

In Fig. 4 we plot the $L=0,1$ Fermi liquid parameters (in units of $\mathrm{fm}^{2}$ ) as a function of the nuclear density (normalized to that of saturated nuclear matter $\rho_{0}=0.16 \mathrm{fm}^{-3}$ ) employing the set of low-energy constants

$$
c_{1}=-0.81 \mathrm{GeV}^{-1}, \quad c_{3}=-3.2 \mathrm{GeV}^{-1}, \quad c_{4}=5.4 \mathrm{GeV}^{-1}, \quad c_{D}=-0.20, \quad c_{E}=-0.205,
$$

which have been used with the Idaho $\mathrm{N}^{3} \mathrm{LO}$ chiral two-nucleon interaction to reproduce the binding energies of $A=3$ nuclei and the triton half-life. We observe that for densities greater than $\rho \simeq \rho_{0} / 4$, both the $L=0$ and $L=1$ Fermi liquid parameters depend approximately linearly on the density, which is not immediately obvious from the analytical expressions given in the previous section. The largest effect on the quasiparticle interaction is a dramatic increase in the isotropic spin- and isospin-independent component, $f_{0}$, which at nuclear matter saturation density is about four times larger than any of the other contributions to the $L=0$ parameters. In fact, both $g_{0}$ and $f_{0}^{\prime}$ remain negligibly small for all densities considered here, while the parameter $g_{0}^{\prime}$ decreases monotonically with the density and reaches the value $-0.3 \mathrm{fm}^{2}$ 


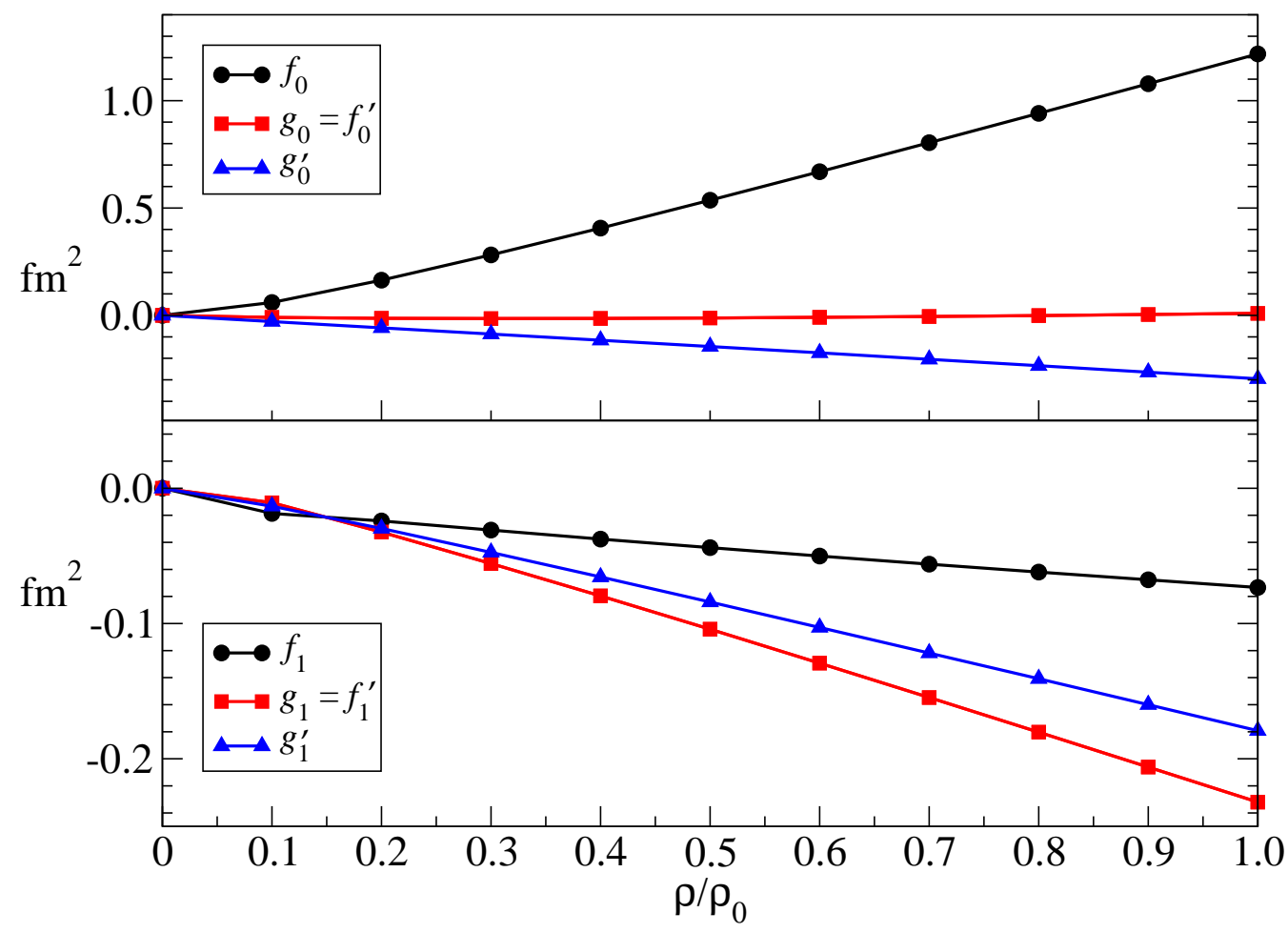

FIG. 4. Density-dependent $L=0,1$ Fermi liquid parameters obtained from the leading-order chiral three-nucleon force with low-energy constants given in eq. (36).

at nuclear matter saturation density $\rho_{0}$. The $L=1$ Fermi liquid parameters all decrease with increasing density. The relatively small change in $f_{1}$ provides only a small downward correction to the quasiparticle effective mass $M^{*}$. This observation, combined with the small change in $f_{0}^{\prime}$ discussed above, indicate that three nucleon forces have a relatively small effect on the nuclear symmetry energy $\beta$. The significant reduction in the parameter $f_{1}^{\prime}$ decreases the nucleon anomalous orbital $g$-factor $\delta g_{l}$ considerably below its empirical value.

In Table \we show the various contributions to the $L=0,1$ Fermi liquid parameters arising from the six topologically-distinct diagrams of Figs. 2 and 3 , These are labeled as $V_{N N}^{\text {med, } i}$ for $i=1, \ldots, 6$. Contributions from the long-range two-pion exchange component of the threebody force are significantly larger than those from the mid- and short-range three-body forces. However, since both $V_{N N}^{\text {med,1 }}$ and $V_{N N}^{\text {med,2 }}$ are medium modifications to one-pion exchange but enter with opposite sign, to a large extent they cancel in all Fermi liquid parameters. This leaves the Pauli-blocked two-pion exchange diagram $V_{N N}^{\text {med,3 }}$ as the dominant contribution, except in the Fermi liquid parameters $g_{0}=f_{0}^{\prime}$ and $f_{1}$ where its effects are surprisingly small. It is 


\begin{tabular}{|c||c|c|c|c|c|c||c|}
\hline \multicolumn{7}{|c|}{$k_{F}=1.33 \mathrm{fm}^{-1}$} \\
\hline & $V_{N N}^{\text {med, } 1}$ & $V_{N N}^{\text {med, } 2}$ & $V_{N N}^{\text {med, } 3}$ & $V_{N N}^{\text {med, } 4}$ & $V_{N N}^{\text {med, } 5}$ & $V_{N N}^{\text {med, } 6}$ & Total \\
\hline \hline$f_{0}\left[\mathrm{fm}^{2}\right]$ & 0.976 & -1.196 & 1.277 & -0.018 & -0.036 & 0.215 & 1.218 \\
\hline$g_{0}=f_{0}^{\prime}\left[\mathrm{fm}^{2}\right]$ & -0.325 & 0.399 & -0.011 & 0.006 & 0.012 & -0.072 & 0.009 \\
\hline$g_{0}^{\prime}\left[\mathrm{fm}^{2}\right]$ & 0.108 & -0.133 & -0.209 & -0.002 & 0.012 & -0.072 & -0.295 \\
\hline \hline$f_{1}\left[\mathrm{fm}^{2}\right]$ & -0.359 & 0.216 & 0.064 & 0.005 & 0 & 0 & -0.073 \\
\hline$g_{1}=f_{1}^{\prime}\left[\mathrm{fm}^{2}\right]$ & 0.120 & -0.072 & -0.278 & -0.002 & 0 & 0 & -0.232 \\
\hline$g_{1}^{\prime}\left[\mathrm{fm}^{2}\right]$ & -0.040 & 0.024 & -0.164 & 0.001 & 0 & 0 & -0.179 \\
\hline \hline
\end{tabular}

TABLE I. Individual contributions to the $L=0,1$ Fermi liquid parameters arising from the six diagrams in Figs. 2 and 3. The contributions are evaluated at nuclear matter saturation density $\rho_{0}=0.16 \mathrm{fm}^{-3}$ corresponding to a Fermi momentum of $k_{F}=1.33 \mathrm{fm}^{-1}$. The values of the low-energy constants of the leading-order chiral three-nucleon force are those given in eq. (36).

instructive to compare the final results to what would be obtained in the chiral limit $\left(m_{\pi} \rightarrow 0\right)$ :

$$
\begin{gathered}
\left(f_{0}\right)^{\chi}=1.287 \mathrm{fm}^{2}, \quad\left(g_{0}\right)^{\chi}=\left(f_{0}^{\prime}\right)^{\chi}=0.136 \mathrm{fm}^{2}, \quad\left(g_{0}^{\prime}\right)^{\chi}=-0.424 \mathrm{fm}^{2} \\
\left(f_{1}\right)^{\chi}=-0.073 \mathrm{fm}^{2}, \quad\left(g_{1}\right)^{\chi}=\left(f_{1}^{\prime}\right)^{\chi}=-0.268 \mathrm{fm}^{2}, \quad\left(g_{1}^{\prime}\right)^{\chi}=-0.203 \mathrm{fm}^{2}
\end{gathered}
$$

which are not very different from those at the physical pion mass.

To assess the scale dependence of the Fermi liquid parameters resulting from the choice of momentum cutoff in the nuclear interaction, we consider next the set of low-energy constants employed in ref. [11] to fit $A=3,4$ binding energies using in addition a low-momentum NN interaction $V_{\text {low-k }}$ defined at the resolution scale $\Lambda=2.1 \mathrm{fm}^{-1}$ :

$$
c_{1}=-0.76 \mathrm{GeV}^{-1}, \quad c_{3}=-4.78 \mathrm{GeV}^{-1}, \quad c_{4}=3.96 \mathrm{GeV}^{-1}, \quad c_{D}=-2.06, \quad c_{E}=-0.625
$$

In Fig. 5 we plot the density-dependent Fermi liquid parameters resulting from the first-order perturbative contribution from the chiral three-nucleon force. Qualitatively, many of the trends observed with the previous set of low-energy constants (eq. (36) ) remain. However, the value of the isotropic spin-independent and isospin-independent Fermi liquid parameter $f_{0}$ is significantly larger. At nuclear matter saturation density, its value of $f_{0}=2.49 \mathrm{fm}^{2}$ is twice as large as that observed in Fig. 4. This repulsion more than compensates for the additional attraction that results from employing low-momentum two-nucleon interactions [1]. The parameters $g_{0}$ and $f_{0}^{\prime}$, which were nearly zero across the wide range of densities shown in Fig. 4, now achieve a value of $-0.24 \mathrm{fm}^{2}$ at saturation density. All of the $L=1$ Fermi liquid parameters 


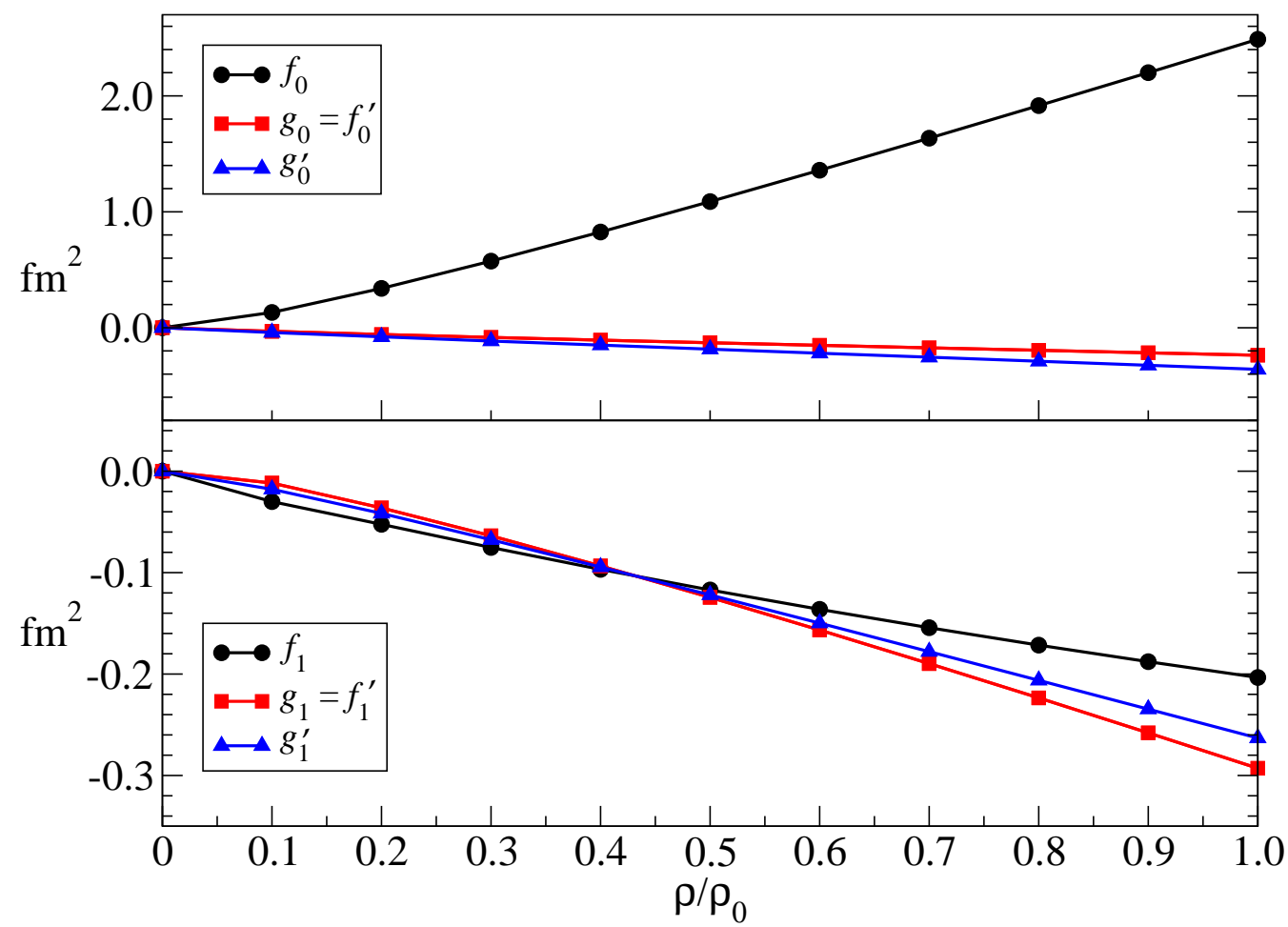

FIG. 5. Density-dependent $L=0,1$ Fermi liquid parameters obtained from the leading-order chiral three-nucleon force with low-energy constants given in eq. (38).

exhibit a similar density dependence, and at saturation density lie within the range $-0.2 \mathrm{fm}^{2}$ to $-0.3 \mathrm{fm}^{2}$. The Fermi liquid parameter $f_{1}$, determining the quasiparticle effective mass $M^{*}$, is twice as large as that obtained using the low-energy constants given in eq. (36) and thus gives rise to a more visible reduction of $M^{*}$.

We display in Table II the decomposition of the Fermi liquid parameters into contributions from the six unique topologies show in Figs. 2 and 3. Although the mid- and short-range components of the three-nucleon force are now larger in magnitude, taken together they largely cancel (in the $L=0$ FLPs) and again give a relatively small contribution to the total sum. The largest differences between the values given in Tables $\Pi$ and $\Pi$ arise from the parameter $c_{3}$. Employing the low-energy constants in eq. (36) except with $c_{3}=-4.78$ would yield the Fermi liquid parameters

$$
\begin{gathered}
f_{0}=2.237 \mathrm{fm}^{2}, \quad g_{0}=f_{0}^{\prime}=-0.150 \mathrm{fm}^{2}, \quad g_{0}^{\prime}=-0.341 \mathrm{fm}^{2} \\
f_{1}=-0.210 \mathrm{fm}^{2}, \quad g_{1}=f_{1}^{\prime}=-0.293 \mathrm{fm}^{2}, \quad g_{1}^{\prime}=-0.265 \mathrm{fm}^{2},
\end{gathered}
$$

which are very close to those in Table $\underline{\text { II. }}$

Finally, we combine these results with previous calculations [1] in which the second-order contribution to the quasiparticle interaction was computed to second order employing chiral 


\begin{tabular}{|c||c|c|c|c|c|c||c|}
\hline \multicolumn{7}{|c|}{$k_{F}=1.33 \mathrm{fm}^{-1}$} \\
\hline & $V_{N N}^{\text {med, } 1}$ & $V_{N N}^{\text {med, } 2}$ & $V_{N N}^{\text {med, } 3}$ & $V_{N N}^{\text {med, } 4}$ & $V_{N N}^{\text {med, } 5}$ & $V_{N N}^{\text {med, } 6}$ & Total \\
\hline \hline$f_{0}\left[\mathrm{fm}^{2}\right]$ & 1.412 & -1.171 & 2.149 & -0.189 & -0.369 & 0.655 & 2.488 \\
\hline$g_{0}=f_{0}^{\prime}\left[\mathrm{fm}^{2}\right]$ & -0.471 & 0.390 & -0.124 & 0.063 & 0.123 & -0.218 & -0.237 \\
\hline$g_{0}^{\prime}\left[\mathrm{fm}^{2}\right]$ & 0.157 & -0.130 & -0.270 & -0.021 & 0.123 & -0.218 & -0.359 \\
\hline \hline$f_{1}\left[\mathrm{fm}^{2}\right]$ & -0.570 & 0.394 & -0.082 & 0.054 & 0 & 0 & -0.203 \\
\hline$g_{1}=f_{1}^{\prime}\left[\mathrm{fm}^{2}\right]$ & 0.190 & -0.131 & -0.333 & -0.018 & 0 & 0 & -0.293 \\
\hline$g_{1}^{\prime}\left[\mathrm{fm}^{2}\right]$ & -0.063 & 0.044 & -0.250 & 0.006 & 0 & 0 & -0.263 \\
\hline \hline
\end{tabular}

TABLE II. Individual contributions to the $L=0,1$ Fermi liquid parameters arising from the six diagrams in Figs. 2 and 3. The contributions are evaluated at nuclear matter saturation density $\rho_{0}=0.16 \mathrm{fm}^{-3}$ corresponding to a Fermi momentum of $k_{F}=1.33 \mathrm{fm}^{-1}$. The values of the low-energy constants of the leading-order chiral three-nucleon force are those given in eq. (38).

and low-momentum NN interactions. The density dependence of the dimensionful Fermi liquid parameters obtained from the low-momentum NN interaction $V_{\text {low-k }}$ at a cutoff scale $\Lambda=$ $2.1 \mathrm{fm}^{-1}$ together with the associated three-nucleon force is shown in Fig. 6 (qualitatively similar results hold also for the unevolved $\mathrm{NN}$ interaction considered in this work). Both the $L=0,1$ components of the spin- and isospin-independent quasiparticle interaction vary strongly with the density, and the inclusion of three-nucleon forces significantly enhances the trend observed in the second-order calculation including two-body forces only [1] (note that in contrast to the current calculation, the results shown in Fig. 3 of ref. [1] did not yet include self-consistent single-particle energies in the denominators of the second-order terms). The parameters associated with the spin- and isospin-dependent components of the quasiparticle interaction, on the other hand, are much more stable to variations in the density (in qualitative agreement with observations made in ref. [14]). In fact, for these components the three $L=0$ terms have a remarkably similar density dependence, and the three $L=1$ terms exhibit this same property.

We now discuss the implications for physical observables. We display in Table III the dimensionless Fermi liquid parameters (obtained by multiplying the dimensionful parameters by the density of states at the Fermi surface $\left.N_{0}=2 M^{*} k_{F} / \pi^{2}\right)$ and the related quasiparticle and bulk nuclear matter properties resulting from calculations including two-nucleon interactions alone as well as including the leading-order chiral three-nucleon force. The former are denoted with superscript $2 N$ while the latter are denoted with $3 N$. The most important feature is a qualitative change of the isotropic spin- and isospin-independent Fermi liquid parameter 


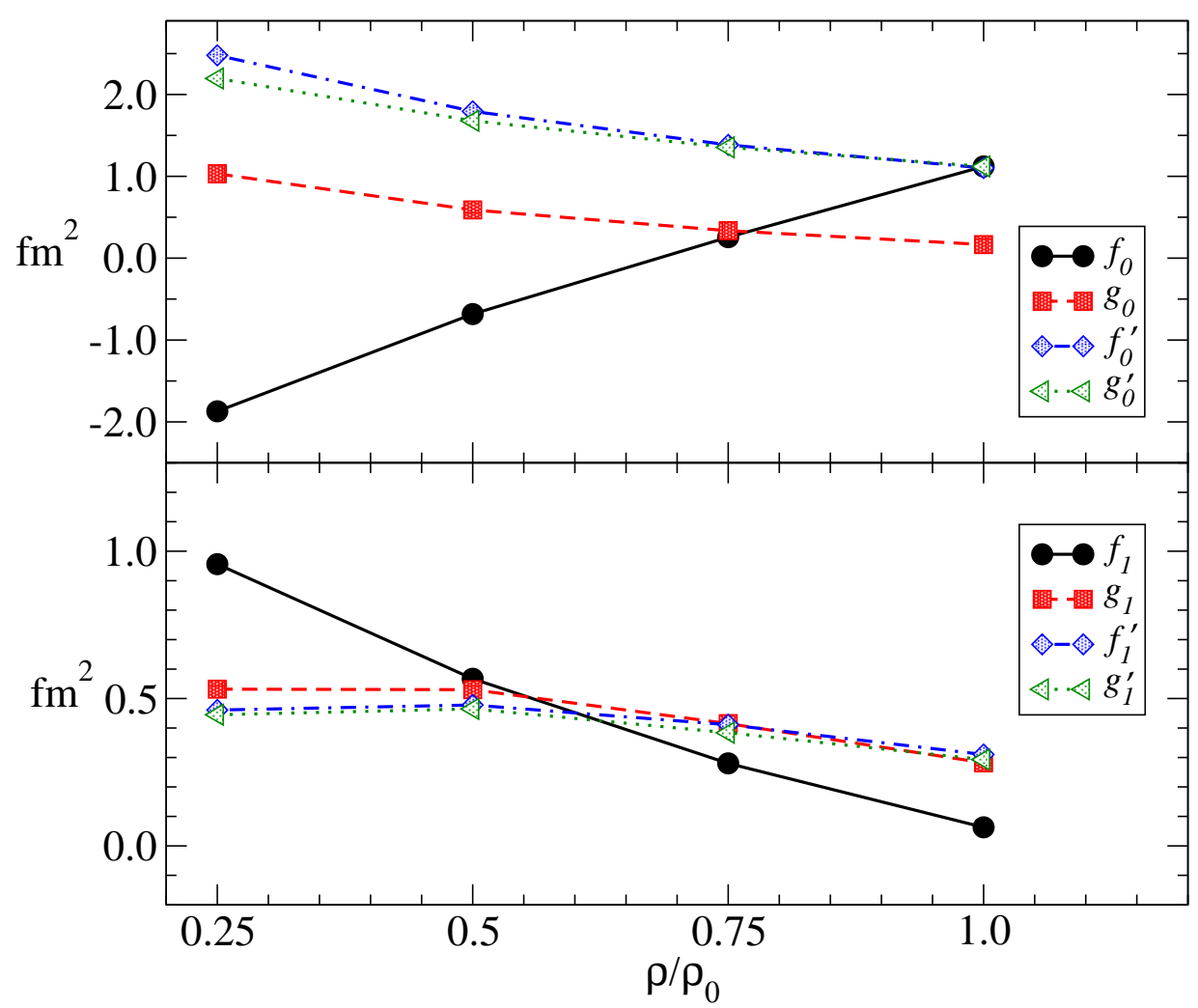

FIG. 6. Density-dependent $L=0,1$ Fermi liquid parameters. Contributions from two-nucleon and three-nucleon forces at the resolution scale $\Lambda=2.1 \mathrm{fm}^{-1}$ are added together.

$F_{0}$, which was large and negative without three-body forces but which now attains a mean value that is positive and relatively small. In fact, the compression modulus $\mathcal{K}$ computed with the low-momentum interaction is now quite large $(\mathcal{K} \simeq 530 \mathrm{MeV})$ compared to values $\mathcal{K}=(200-300) \mathrm{MeV}$ extracted from studies of giant monopole resonances in heavy nuclei [3739]. Given that the use of low-momentum two- and three-body interactions yields a quite good description of nuclear matter saturation already in (approximate) second order perturbative calculations [11, 13], it is likely that the large value of $F_{0}$ obtained with $V_{\text {low-k }}$ in the present work results from the absence of second-order contributions from three-body forces. Aside from the large uncertainty in the value of $F_{0}$, nearly all the other Fermi liquid parameters now exhibit much less scale dependence. This feature has also been observed in previous calculations [11, 13], where it was found that including three-nucleon forces and higher-order corrections in perturbation theory dramatically reduced the scale dependence of the nuclear matter equation of state. The effective mass $M^{*}$ and the nuclear symmetry energy $\beta$ receive relatively small corrections from three-body forces at this order and to within errors remain in agreement with their empirical values. The observable that is not well described in this calculation is the anomalous orbital $g$-factor $\delta g_{l}$ which remains considerably below the value extracted from 


\begin{tabular}{|l||c|c|c|c||c|c|c|c||c|c|c|c|}
\hline & $F_{0}$ & $G_{0}$ & $F_{0}^{\prime}$ & $G_{0}^{\prime}$ & $F_{1}$ & $G_{1}$ & $F_{1}^{\prime}$ & $G_{1}^{\prime}$ & $M^{*} / M_{N}$ & $\mathcal{K}[\mathrm{MeV}]$ & $\beta[\mathrm{MeV}]$ & $\delta g_{l}$ \\
\hline \hline$V_{\mathrm{N} 3 \mathrm{LO}}^{2 N}$ & -1.64 & 0.35 & 1.39 & 1.59 & -0.13 & 0.50 & 0.58 & 0.47 & 0.96 & -150 & 31 & 0.12 \\
\hline$V_{\text {low }-\mathrm{k}}^{2 N}$ & -1.98 & 0.58 & 1.94 & 2.14 & 0.38 & 0.83 & 0.87 & 0.80 & 1.13 & -190 & 32 & 0.07 \\
\hline \hline$V_{\text {N3LO }}^{3 N}$ & -0.15 & 0.35 & 1.36 & 1.19 & -0.22 & 0.21 & 0.28 & 0.24 & 0.93 & 200 & 31 & 0.09 \\
\hline$V_{\text {low }-\mathrm{k}}^{3 N}$ & 1.48 & 0.22 & 1.45 & 1.48 & 0.08 & 0.37 & 0.41 & 0.39 & 1.03 & 530 & 29 & 0.05 \\
\hline
\end{tabular}

TABLE III. Dimensionless Fermi liquid parameters obtained by summing the second-order contribution from the Idaho $\mathrm{N}^{3} \mathrm{LO}$ potential and low-momentum $\mathrm{NN}$ interactions $V_{\text {low }-\mathrm{k}}\left(\Lambda=2.1 \mathrm{fm}^{-1}\right)$ at $k_{F}=1.33 \mathrm{fm}^{-1}$. Hartree-Fock self-energy insertions, as parameterized in ref. [1], are included in the second-order diagrams.

giant dipole resonances of nuclei. This quantity, however, is particularly sensitive to the value of $F_{1}$ and the quasiparticle effective mass (see eq.(5) ), and therefore even a moderate decrease of the nucleon effective mass $M^{*}$ due to higher-order perturbative corrections could remedy the situation. We summarize below the results for selected nuclear observables, including also theoretical uncertainties estimated by choosing different resolution scales and low-energy constants:

$$
\begin{array}{rlll}
\text { Effective mass : } & \frac{M^{*}}{M_{N}}=0.98 \pm 0.05, & {[0.7-1.0]} \\
\text { Anomalous orbital } g \text {-factor: } & \delta g_{l}= & 0.07 \pm 0.02, & {[0.20-0.26]} \\
\text { Compression modulus : } & \mathcal{K}=(370 \pm 160) \mathrm{MeV}, & {[200-300] \mathrm{MeV}} \\
\text { Isospin asymmetry energy : } \quad \beta= & (30 \pm 1) \mathrm{MeV}, & {[30-36] \mathrm{MeV}} \\
\text { Spin-isospin response : } & g_{N N}^{\prime}= & 0.55 \pm 0.03 & {[0.6-0.7],}
\end{array}
$$

where the quantities in the last column are estimates of the empirical values (see Section IA). A final interesting feature of the three-nucleon force is that the contributions to the $L=1$ Fermi liquid parameters are all negative, with the result that the quasiparticle interaction at the Fermi surface appears to be short ranged.

\section{CONCLUSION}

In this work we have performed the first systematic inclusion of the $\mathrm{N}^{2} \mathrm{LO}$ chiral threenucleon force in calculations of the quasiparticle interaction in nuclear matter. Analytical expressions for the $L=0,1$ Fermi liquid parameters were derived, and numerical results have been extracted for two different sets of low-energy constants $\left(c_{E}, c_{D}\right.$ and $\left.c_{1,3,4}\right)$ corresponding to 
two choices of resolution scale $\Lambda=(500,414) \mathrm{MeV}$ for the two- and three-nucleon interactions. In comparison to calculations employing two-nucleon forces only, we find a significantly smaller dependence of the Fermi liquid parameters on the choice of this cutoff scale. At this order in the perturbative calculation, the bulk properties of nuclear matter and the dynamical features of quasiparticles are in general well described. In particular, the compression modulus $\mathcal{K}$ of nuclear matter attains a reasonable value only with the inclusion of three-nucleon correlations, which introduce large additional repulsion in the effective interaction. Although an analogous effect has already been observed in calculations of the nuclear matter equation of state, the fact that the three-body force gives a disproportionately large contribution to $F_{0}$, compared to all other Fermi liquid parameters, comes as a surprise. The remaining contributions to the $L=0,1$ Fermi liquid parameters from the chiral three-nucleon force are (essentially) all negative. This results in diminished $L=1$ Landau parameters and an effective interaction of apparent short range. A remaining unresolved problem concerns the description of the anomalous orbital $g$ factor $\delta g_{l}$ (which arises from interactions with the nuclear medium). In the present work we find corrections to free space orbital $g$-factor that are much too small, a feature which mainly results from a large nucleon effective mass $\left(M^{*} \simeq M_{N}\right)$. The role played by three-nucleon force contributions beyond the Hartree-Fock approximation and the effect of the subleading $\left(\mathrm{N}^{3} \mathrm{LO}\right)$ chiral three-body force [36] remain topics of interest for future calculations. A possible strategy is to represent three-nucleon force contributions in terms of density-dependent twobody forces [35] and to employ these in second-order calculations. We have verified that treating three-nucleon force contributions in this approximation gives good agreement with their exact evaluation at first order.

We thank A. Schwenk for helpful discussions. 
[1] J. W. Holt, N. Kaiser, and W. Weise, Nucl. Phys. A870-871 (2011) 1.

[2] S. K. Bogner, T. T. S. Kuo, and A. Schwenk, Phys. Rept. 386 (2003) 1.

[3] S. K. Bogner, R. J. Furnstahl and A. Schwenk, Prog. Part. Nucl. Phys. 65 (2010) 94.

[4] O. Sjöberg, Ann. Phys. 78 (1973) 39.

[5] O. Sjöberg, Nucl. Phys. A209 (1973) 363.

[6] W. H. Dickhoff, A. Faessler, H. Müther, and S. S. Wu, Nucl. Phys. A405 (1983) 534.

[7] S. O. Bäckman, G. E. Brown, and J. A. Niskanen, Phys. Rept. 124 (1985) 1.

[8] J. W. Holt, G. E. Brown, J. D. Holt and T. T. S. Kuo, Nucl. Phys. A785 (2007) 322.

[9] S. Babu and G.E. Brown, Ann. Phys. 78 (1973) 1.

[10] S. Fritsch, N. Kaiser and W. Weise, Nucl. Phys. A750 (2005) 259.

[11] S. K. Bogner, A. Schwenk, R. J. Furnstahl, and A. Nogga, Nucl. Phys. A763 (2005) 59.

[12] L.-W. Siu, J. W. Holt, T. T. S. Kuo and G. E. Brown, Phys. Rev. C 79 (2009) 054004.

[13] K. Hebeler, S. K. Bogner, R. J. Furnstahl, A. Nogga and A. Schwenk, Phys. Rev. C 83 (2011) 031301.

[14] N. Kaiser, Nucl. Phys. A768 (2006) 99.

[15] D. R. Entem and R. Machleidt, Phys. Rev. C 68 (2003) 041001(R).

[16] E. Epelbaum, Prog. Part. Nucl. Phys. 57 (2006) 654.

[17] L. D. Landau, Sov. Phys. JETP, 3 (1957) 920; 5 (1957) 101; 8 (1959) 70.

[18] A. B. Migdal, Theory of Finite Fermi Systems and Applications to Atomic Nuclei (Interscience, New York, 1967).

[19] G. Baym and C. Pethick, Landau Fermi-Liquid Theory (Wiley \& Sons, New York, 1991).

[20] P. Nozières and D. Pines, The theory of quantum liquids (Perseus, Cambridge, Massachusetts, 1999).

[21] S.-O. Bäckman, O. Sjöberg and A. D. Jackson, Nucl. Phys. A321 (1979) 10.

[22] P. Haensel and A. J. Jerzak, Phys. Lett. B 112 (1982) 285.

[23] E. Olsson, P. Haensel and C. J. Pethick, Phys. Rev. C 70 (2004) 025804.

[24] S. Bacca, K. Hally, C. J. Pethick and A. Schwenk, Phys. Rev. C 80 (2009) 032802.

[25] J. P. Jeukenne, A. Lejeuene and C. Mahaux, Phys. Rept. 25 (1976) 83.

[26] W. Zuo, I. Bombaci and U. Lombardo, Phys. Rev. C 60 (1999) 024605.

[27] P. Danielewicz, Nucl. Phys. A727 (2003) 233.

[28] A. W. Steiner, M. Prakash, J. M. Lattimer, and P. J. Ellis, Phys. Rept. 411 (2005) 325. 
[29] C. Gaarde, Nucl. Phys. A396 (1983) 127c.

[30] T. Ericson and W. Weise, Pions and Nuclei (Clarendon Press, Oxford, 1988).

[31] T. Suzuki and H. Sakai, Phys. Lett. B455 (1999) 25.

[32] R. Nolte, A. Baumann, K. W. Rose and M. Schumacher, Phys. Lett. B173 (1986) 388.

[33] M. C. M. Rentmeester, R. G. E. Timmermans and J. J. de Swart, Phys. Rev. C 67 (2003) 044001.

[34] D. Gazit, S. Quaglioni, and P. Navrátil, Phys. Rev. Lett. 103 (2009) 102502.

[35] J.W. Holt, N. Kaiser and W. Weise Phys. Rev. C 81 (2010) 024002.

[36] V. Bernard, E. Epelbaum, H. Krebs, U.-G. Meiner, Phys. Rev. C 77, 064004 (2008); arXiv:1108.3816.

[37] J. P. Blaizot, Phys. Rept. 64 (1980) 171.

[38] D. H. Youngblood, H. L. Clark, and Y.-W. Lui, Phys. Rev. Lett. 82 (1999) 691.

[39] M. V. Stoitsov, P. Ring and M. M. Sharma, Phys. Rev. C 50 (1994) 1445. 